\title{
Olfactory Region
}

National Cancer Institute

\section{Source}

National Cancer Institute. Olfactory Region. NCI Thesaurus. Code C98765.

The area of mucosa in the nose lined by olfactory epithelium and containing olfactory glands. 